MODELING, IDENTIFICATION AND CONTROL, 1994, VOL. 15, NO. 3, 191-203

doi:10.4173/mic.1994.3.9

\title{
The role of fuzzy logic in modeling, identification and control
}

\author{
LOTFI A. ZADEH $\dagger$ \\ Keywords: Fuzzy logic; fuzzy control; fuzzy modeling.
}

\begin{abstract}
In the nearly four decades which have passed since the launching of the Sputnik, great progress has been achieved in our understanding of how to model, identify and control complex systems. However, to be able to design systems having high MIQ (Machine Intelligence Quotient), a profound change in the orientation of control theory may be required. More specifically, what may be needed is the employment of soft computing - rather than hard computing -in systems analysis and design. Soft computing - unlike hard computing - is tolerant of imprecision, uncertainty and partial truth.

At this juncture, the principal constituents of soft computing are fuzzy logic, neurocomputing and probabilistic reasoning. In this paper, the focus is on the role of fuzzy logic. The basic ideas underlying fuzzy logic and its applications to modeling, identification and control are described and illustrated by examples. The role model for fuzzy logic is the human mind.
\end{abstract}

\section{Introduction-A personal perspective}

The origins of modern control theory go back to the days of World War II when the analysis and design of servomechanisms played a central role in the conception and construction of electromechanical systems which could transform low-power inputs into high-power outputs.

As a student at MIT - and later a professor at Columbia University-I witnessed the evolution of control theory from a simplistic, frequency-based theory of linear control systems into a mathematically sophisticated theory of linear/nonlinear systems based on the theory of differential equations and dynamical systems.

It was, above all, the advent of the space age in 1957 that changed the orientation and spirit of control theory. The competition in space-and the challenges posed by the complexity of control problems related to the guidance of space vehicles - attracted to control theory a number of prominent mathematicians, most notably L. S. Pontryagin in the USSR and R. E. Bellman in the United States. As a result, the level of mathematical sophistication of the theory began to grow very rapidly, swinging the pendulum all the way from the low-brow approaches of the forties and fifties to the high-brow mathematical formalism of the seventies, eighties and nineties.

In the fifties and early sixties, my interests centred not on control but on a more general theory - system theory - a theory which was aimed at the development of a

Received 31 March 1994.

†Computer Science Division and the Electronics Research Laboratory, Department of EECS, University of California, Berkeley, CA 94720; Tel: 510-642-4959; Fax: 510-642-8271, 510642-5775; Email: zadeh@ cs.berkeley.edu.

Research supported in part by the BISC Program, NASA Grant NCC 2-275, EPRI Agreement RP 8010-34 and MICRO State Program No. 92-180. 
better understanding of how abstract systems behave and how they could be designed to perform in a specified way.

As a mathematically oriented system theorist, I had been conditioned to believe that the analytical tools based on set theory and two-valued logic were all that was needed to build a framework for a precise, rigorous and effective body of concepts and techniques for the analysis of almost any kind of man-made or natural system, including control systems. Then, in 1961-1963, in the course of writing a book on system theory with C. A. Desoer, I began to feel that highly complex systems - typified by economic and biological systems--cannot be dealt with effectively by the use of conventional approaches. My feeling derived, in the main, from a realization that system description languages based on classical mathematics are not sufficiently expressive to serve as a means of characterization of complex input-output relations in an environment of imprecision and uncertainty.

The culprit as I saw it was the universally made assumption that classes have sharply defined boundaries. They do in classical mathematics, but in the real world that we live in the opposite is the case, that is, almost all classes are fuzzy in the sense that the transition from membership to nonmembership in such classes is gradual rather than abrupt. Accepting this fact, the obvious thing to do is to assume that membership in a class is a matter of degree. This assumption is the genesis of the theory of fuzzy sets.

After I wrote my first paper on fuzzy sets in 1965, my aims as a system theorist underwent a marked shift. I came to the conclusion that not just control theory and systems analysis, but, more generally, most or all scientific methodologies will have to undergo a critical reexamination and move toward the replacement of their crisp foundations with foundations based on fuzzy set theory and fuzzy logic (Zadeh 1971, 1972), aiming at greater generality and better approximation to reality. In the realm of control, in particular, the replacement of crisp logic with fuzzy logic could make it possible to design systems with a much higher MIQ (Machine Intelligence Quotient) compared to those which can be designed by traditional methods. In what foliows, I will attempt to describe very briefly the basis for my conclusion.

\section{Fuzzy logic, linguistic variables and granulation}

Fuzzy logic has been and still is somewhat controversial. In part, the controversy relates to the fact that the term fuzzy logic is used in two different senses. In a narrow sense, fuzzy logic (FLn) is a logical system which aims at a formalization of approximate reasoning. As such, it is rooted in multivalued logic but its agenda is quite different from that of traditional multivalued logical systems, e.g., Lukasiewicz's logic. In this connection, it should be noted that many of the concepts which account for the effectiveness of fuzzy logic as a logic of approximate reasoning are not a part of traditional multivalued logical systems. Among these are the concepts of a linguistic variable, canonical form, fuzzy rule, fuzzy graph, fuzzy quantifiers and such modes of reasoning as interpolative reasoning, syllogistic reasoning and dispositional reasoning.

In a broad sense, fuzzy logic (FLw) is almost synonymous with fuzzy set theory. Fuzzy set theory is much broader than fuzzy logic in its narrow sense and contains the latter as one of its branches. Among the other branches of fuzzy set theory are fuzzy arithmetic, fuzzy mathematical programming, fuzzy topology, fuzzy graph theory, possibility theory, etc. What is important to recognize is that any crisp theory can be fuzzified by generalizing the concept of a set within that theory to the concept of a fuzzy set. Indeed, it is very likely that eventually most theories will be fuzzified in this way. As 
was alluded to earlier, the impetus for transition from a crisp theory to a fuzzy one derives from the fact that both the generality of a theory and its applicability to real world problems are substantially enhanced by replacing the concept of a set with that of a fuzzy set.

Today, the growing tendency is to use the term fuzzy logic $(\mathrm{FL})$ in its broad sense. In part this reflects the fact that fuzzy logic sounds more euphonious than fuzzy set theory.

A concept which plays a central role in the applications of fuzzy logic is that of a linguistic variable (Zadeh 1973, 1975). A linguistic variable, as its name suggests, is a variable whose values are words rather than numbers. For example, age is a linguistic variable if its values are: young, old, not very old, etc. Each linguistic value is interpreted as a label of a fuzzy set in its universe of discourse and each such set, e.g., old, is defined by its membership function, $\mu_{\text {old }}(u)$, which associates with each numerical value, $u$, of age the degree to which $n$ fits one's subjective, context-dependent perception of the meaning of old. The grade of membership is assumed to take values in the interval $[0,1]$.

In current practice, in most applications to control, the membership functions of linguistic values are assumed to be trapezoidal or triangular in shape (Fig. 1). The number of linguistic values is usually in the range of three to seven, and the values are labeled positive small (PS), negative large (NL), zero (Z), etc.

There is a basic aspect of the concept of a linguistic variable which is at the heart of its utility. Specifically, consider a linguistic variable such as age whose linguistic values are young, middle-aged, and old, with young defined by a membership function such as shown in Fig. 2.

Clearly, a numerical value such as 25 is simpler than the function young. But young represents a choice of one out of three possible values whereas 25 is a choice of one out of, say, 100 values. The point of this simple example is that the use of linguistic values may be viewed as a form of data compression. It is suggestive to refer to this form of data compression as fuzzy granulation or simply granulation.

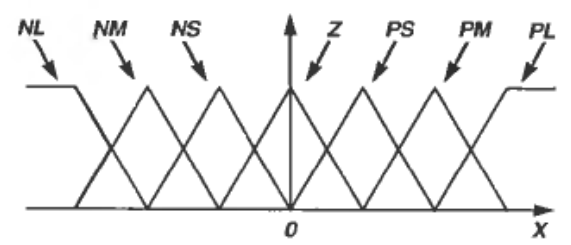

Figure 1. Triangular linguistic values.
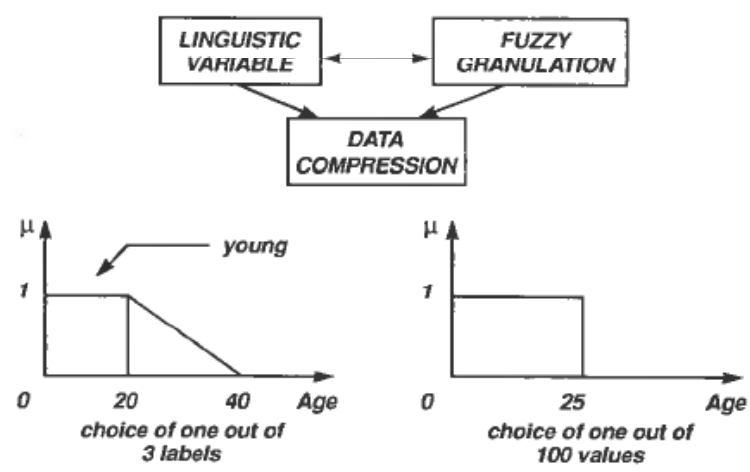

Figure 2. Granulation and data compression. 


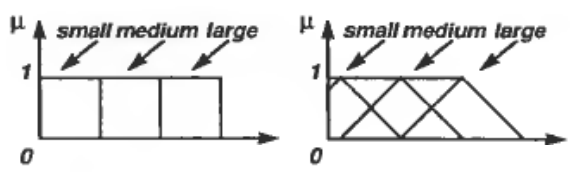

Figure 3. Quantization vs. granulation.

The same effect can be achieved, of course, by conventional quantization. But in the case of quantization, the values are crisp intervals whereas in the case of granulation the values are overlapping fuzzy intervals (Fig. 3), resulting in a gradual rather than abrupt transition from one value to another.

The graduality of transition mimics the way in which humans perceive linguistic values. More importantly - as will be seen later-it provides a basis for interpolative reasoning. Such reasoning plays a central role in learning from experience.

\section{Fuzzy rules}

In fuzzy logic, the concept of a linguistic variable serves as a point of departure for the construction of a language, referred to as FDCL (Fuzzy Dependency and Command Language), which provides a framework for the description of imprecise dependencies and commands through the use of fuzzy if-then rules or simply fuzzy rules. In what follows, we shall sketch some of the basic ideas underlying FDCL.

Like any language, FDCL is characterized by its syntax and semantics. The syntax and semantics of FDCL define, respectively, the form of rules and their meaning.

Simple examples of the rules in FDCL are the following.

(a) if pressure is high then volume is small

(b) if pressure is high and temperature is low then volume is very small

(c) if pressure is high then lower temperature slightly

(d) if pressure is high then volume is small unless temperature is high

(e) if pressure is high then usually volume is small.

In these examples, pressure, volume and temperature are linguistic variables and small, low and high are their linguistic values. All of the rules except $(c)$ represent dependencies, with $(c)$ representing a command. All of the rules except $(d)$ and $(e)$ are categorical. Rules $(d)$ and $(e)$ are qualified, with $(d)$ qualified through an exception and $(e)$ through usuality. (e) exemplifies what is referred to as a dispositional rule. All of the rules except for $(b)$ involve a single variable in the antecedent. A real-world example of rules used in Honda's fuzzy logic transmission is shown in Fig. 4.

Assuming that small, low, high, usually and whatever other linguistic values may be involved are defined by their membership functions, as in Fig. 4, a question of semantics is: What is the meaning of a rule? And, more generally: What is the meaning of a collection of rules? We shall examine these questions very briefly in the context of basic generic rules of the form

$$
\text { if } X \text { is } A \text { then } Y \text { is } B \text {, }
$$

where $A$ and $B$ are linguistic values of $X$ and $Y$, respectively.

In fuzzy logic, the answers to these questions are not unique. In what has become a standard interpretation, (1) is interpreted as a fuzzy (elastic) constraint on the variables $X$ and $Y$. More specifically, with $\rightarrow$ representing translation, we have

$$
\text { if } X \text { is } A \text { then } Y \text { is } B \rightarrow(X, Y) \text { is } A \times B \text {, }
$$




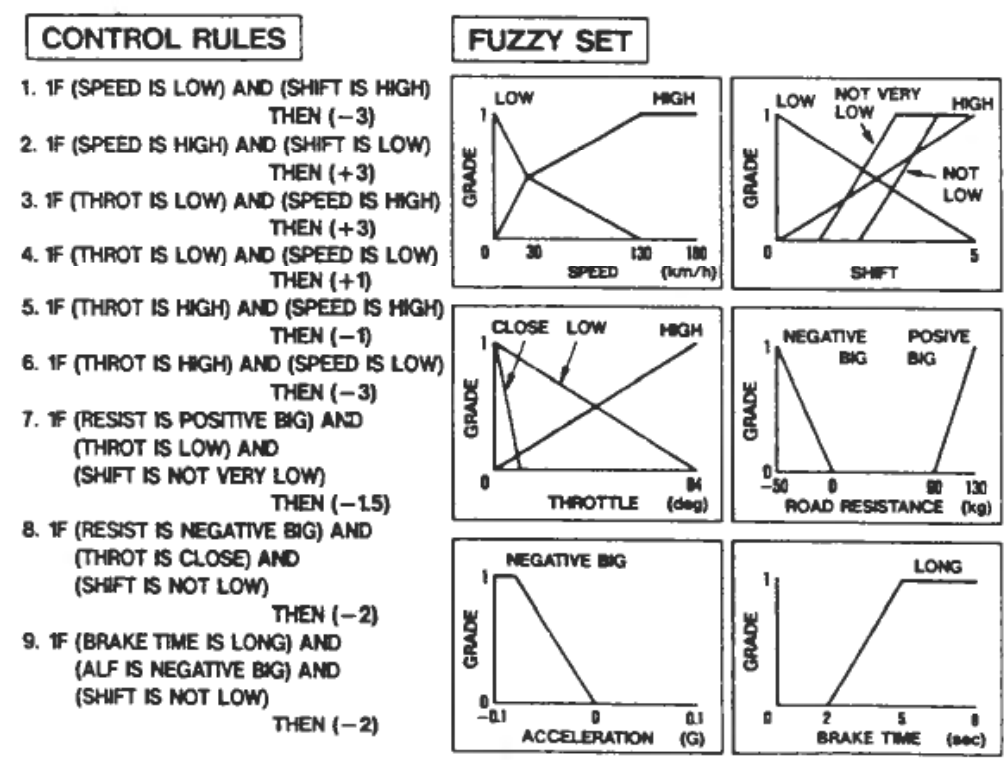

Figure 4. Fuzzy rules employed in Honda's fuzzy logic transmission.

where $\mathrm{A} \times \mathrm{B}$ is the cartesian product of $A$ and $B . A \times B$ is defined by

$$
\mu_{A \times B}(u, v)=\mu_{A}(u) \wedge \mu_{B}(v), u \in U, v \in V,
$$

where $\mu_{A}$ and $\mu_{B}$ are the membership functions of $\mathrm{A}$ and $\mathrm{B}$, respectively; $\mathrm{U}$ and $\mathrm{V}$ are the universes of discourse of $X$ and $Y$; and $\wedge$ is the operation of conjunction, which is usually defined as min but, more generally, can be any t-norm (Zimmermann 1990).

More generally, if the dependence of $Y$ on $X$ is characterized by a collection of $n$ rules of the form (1), that is:

$$
\text { if } X \text { is } A_{i} \text { then } Y \text { is } B_{i}, i=1, \ldots, n \text {, }
$$

then the meaning of the collection in question is defined by

$$
\text { if } X \text { is } A_{i} \text { then } Y \text { is } B_{i}, i=1, \ldots, n \rightarrow(X, Y) \text { is } A_{1} \times B_{1}+\ldots+A_{n} \times B_{n} \text {, }
$$

where + denotes disjunction. Usually the disjunction is defined as max but, more generally, could be any t-conorm. For convenience, the right-hand member of (4) can be written as

$$
(X, Y) \text { is } \Sigma_{i} A_{i} \times B_{i} \text {. }
$$

If $f$ is a crisp function of $X, Y=f(\mathrm{X})$, the expression

$$
f^{*}=\Sigma_{i} A_{i} \times B_{i}
$$

constitutes the fuzzy graph of $f$ (Zadeh 1971, 1973/74a, 1974b/76). More generally, if $Y=f\left(X_{1}, \ldots, X_{n}\right)$ is defined via $n$ fuzzy rules of the form

$$
\mathrm{Y} \text { is } B_{i} \text { if } X_{1} \text { is } A_{i}^{1} \text { and } \ldots \text { and } X_{k} \text { is } A_{i}^{k}, i=1, \ldots, n
$$

then the fuzzy graph, $f^{*}$, is expressed as

$$
f^{*}=\Sigma_{i} B_{i} \times A_{i}^{1} \times \ldots \times A_{i}^{k}, i=1, \ldots, n .
$$

As shown in Fig. 5, $f^{*}$ may be interpreted as a coarse relational approximation to $f$. 


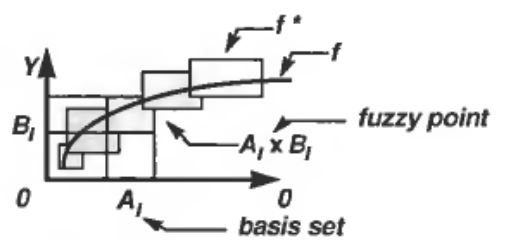

Figure 5. Fuzzy graph approximation to $f$.

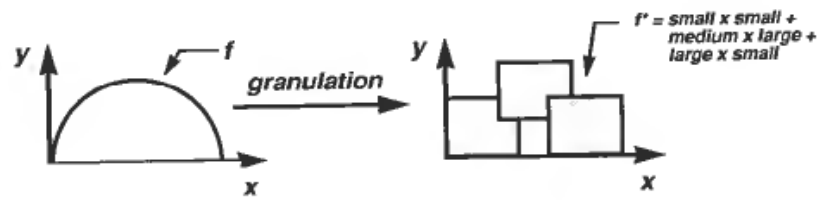

Figure 6. Granulation and data compression.

What is important to note is that $f^{*}$ serves to represent the dependence of $\mathrm{Y}$ on $\mathrm{X}$ in a data-compressed form. For example, in the case of the function shown in Fig. 6, the fuzzy graph

$$
f^{*}=\text { small } \times \text { small }+ \text { medium } \times \text { large }+ \text { large } \times \text { small }
$$

may be interpreted as a compressed representation of $f$.

Manipulation of compressed representations expressed as fuzzy graphs is the province of the calculus of fuzzy graphs (CFG) (Zadeh 1994). Most of the applications of fuzzy logic in the realms of systems analysis and control are in reality applications of CFG.

The importance of CFG derives from the fact that it provides a simple and yet effective way of representing imprecise dependencies. What should be stressed is that CFG does not replace the traditional calculi of differential equations, difference equations, matrices, etc. Rather, it adds to the collection of tools for systems analysis a body of concepts and techniques which are effective in dealing with dependencies which are imprecise to begin with or can be treated imprecisely to achieve tractability, robustness or low solution cost.

\section{Fuzzy modeling and control}

In an idealized sense, a system $S$ has a model $\mathrm{M}(\mathrm{S})$ if $\mathrm{M}(\mathrm{S})$ makes it possible to predict the response of $S$ to any given input. In reality, the response of $\mathrm{M}(\mathrm{S})$ is usually an approximation to the response of $S$ - an approximation which holds for a restricted set of inputs.

In traditional approaches to systems analysis, $\mathrm{M}(\mathrm{S})$ is generally represented as a collection of differential or difference equations. In fuzzy-logic-based approaches, $\mathrm{M}(\mathrm{S})$ is represented as a collection of fuzzy if-then rules or, more particularly, as a collection of fuzzy rules of the form (6). In this sense, $M(S)$ is a fuzzy model if through the use of the calculi of fuzzy rules or fuzzy graphs the knowledge of $\mathrm{M}(\mathrm{S})$ makes it possible to predict the response of $\mathrm{S}$ to any given fuzzy input. 


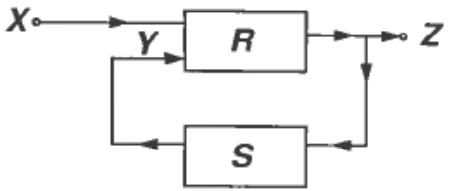

Figure 7. Example of a fuzzy model.

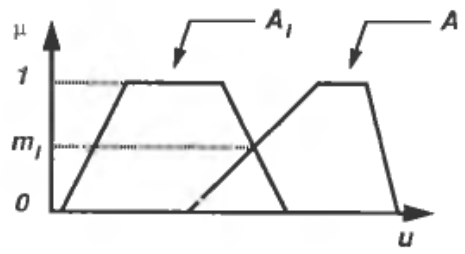

Figure 8. The meaning of the degree of match between $A$ and $A_{i}$.

An example of a fuzzy model is shown in Fig. 7. In this model, R and S are assumed to be characterized by the fuzzy rules

$$
\begin{aligned}
& \left\{\begin{array}{l}
Z \mathrm{Z} \text { is large if } \mathrm{X} \text { is small and } \mathrm{Y} \text { is medium } \\
\mathrm{Z} \text { is small if } \mathrm{X} \text { is medium and } \mathrm{Y} \text { is not very small }
\end{array}\right. \\
& S\left\{\begin{array}{l}
\mathrm{Y} \text { is small if } \mathrm{Z} \text { is large } \\
\mathrm{Y} \text { is medium if } \mathrm{Z} \text { is not very large } \\
\ldots \ldots \ldots \ldots \ldots
\end{array}\right.
\end{aligned}
$$

The calculus of fuzzy graphs provides a computationally simple method of computing the response of $S$ to any given fuzzy input through the use of fuzzy interpolation. The basis for the computation in question is the interpolation rule

$$
\begin{gathered}
(X, Y) \text { is } \Sigma_{i} A_{i} \times B_{i} \\
\frac{X \text { is } A}{Y \text { is } \Sigma_{i} m_{i} \wedge B_{i}}
\end{gathered}
$$

where $m_{i}$ represents the degree to which the value of $X, A$, matches the antecedent of $i$ th rule. More specifically

$$
m_{i}=\sup _{u}\left(\mu_{A}(u) \wedge \mu_{A_{i}}(u)\right)
$$

where the supremum is taken over all $u \in U$ (Fig. 8).

The interpolation rule (7) is a derivative of the compositional rule of inference (Zadeh 1973); it was described in Zadeh (1974a, 1974/76b) and was employed independently by Mamdani and Assilian in their seminal work on fuzzy control (Mamdani 1975). Today, the interpolation rule and its variants play a central role in most of the applications of fuzzy logic to control and systems analysis.

In the foregoing discussion, we have tacitly restricted our attention to static fuzzy systems. However, as shown in some of our early work (Zadeh 1971, 1974), the method in question can readily be extended to dynamic fuzzy systems. 
More specifically, let $X_{t}, Y_{t}$ and $S_{t}$ denote the fuzzy input, fuzzy output and fuzzy state at time $t, t=0,1,2, \ldots$, of a finite-state fuzzy system $S$. Then, the state equations of $S$ may be expressed as

$$
\begin{aligned}
& S_{t+1}=f\left(S_{t}, X_{t}\right), t=0,1,2, \ldots \\
& Y_{t}=g\left(S_{t}, X_{t}\right) .
\end{aligned}
$$

Expressed in FDCL, the state equations can be represented as a collection of fuzzy rules of the form

$$
\begin{aligned}
& \text { if } X_{t} \text { is } A_{i} \text { and } S_{t} \text { is } B_{i} \text { then } S_{t+1} \text { is } C_{i}, i=1, \ldots, n \\
& \text { if } X_{t} \text { is } A_{i} \text { and } S_{t} \text { is } B_{i} \text { then } Y_{t} \text { is } D_{i},
\end{aligned}
$$

where $A_{i}, B_{i}$ and $C_{i}$ are linguistic values of $X_{t}, S_{t}$ and $Y_{t}$, respectively.

Equivalently, the state equations can be represented as fuzzy graphs

$$
\begin{gathered}
f^{*}=\Sigma_{i} A_{i} \times B_{i} \times C_{i} \\
g^{*}=\Sigma_{i} A_{i} \times B_{i} \times D_{i} .
\end{gathered}
$$

As a simple illustration, assume that the linguistic values of $X_{t}, S_{t}$ and $Y_{t}$ are $\mathrm{S}$ (small), $M$ (medium) and $\mathrm{L}$ (large), respectively, with the understanding that the membership functions of $\mathrm{S}, \mathrm{M}$ and $\mathrm{L}$ are not necessarily the same for $X_{t}, S_{t}$ and $Y_{t}$. Expressed in tabular form, the state equation may have, as an example, the form shown in Table 1.

In cases where the spaces of states, inputs and outputs are continua rather than finite sets it may be convenient to represent $S_{t+1}$ in incremental form, that is,

$$
S_{t+1}=S_{t}+\delta S_{t} \text {. }
$$

As an illustration, in the case of an inverted pendulum, $S_{t}=\left(\theta_{t}, \dot{\theta}_{t}\right)$, where $\theta_{t}$ is the vertical inclination and $\theta_{t}$ is the time-derivative of $\theta_{t}$, with both $\theta_{t}$ and $\theta_{t}$ interpreted as linguistic variables with linguistic values Z (zero), PS (positive small), NS (negative small), etc. In this case, an entry in the state transition table may be expressed as a fuzzy rule exemplified by

$$
\text { if } S_{t} \text { is }(Z, P S) \text { and } X_{t} \text { is } P S \text { then } \delta S_{t} \text { is }(N S, Z) \text {. }
$$

Knowledge of a fuzzy model of $\mathrm{S}$ in a direct or incremental form provides a basis for the formulation and solution of various types of generic control problems centring on $\mathrm{S}$. One such problem is the following.

Table 1. State equations of a finite-state fuzzy system.

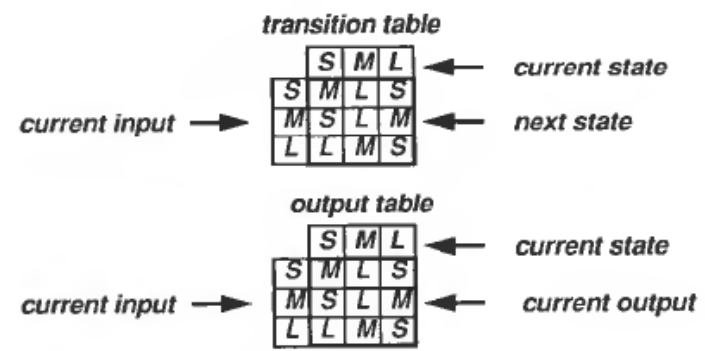


Given an initial state $S_{I}$ and a goal state $S_{G}$ in the state space of $S$ find a policy function $\pi$,

$$
X_{t}=\pi\left(S_{t}\right)
$$

which would take $S$ from the initial state at time $t=0$ to a state at time $t=N$ which is nearest to $S_{G}$.

Problems of this type can be solved through the use of fuzzy dynamic programming (Bellman and Zadeh 1970). More specifically, assume that the error at time $N$ is defined as the distance, $d$, between $S_{N}$ and $S_{G}$, i.e.,

$$
e_{N}\left(S_{N}\right)=d\left(S_{N}, S_{G}\right)
$$

Using backward iteration, we can write

$$
\begin{aligned}
e_{N-1}\left(S_{N-1}\right)= & \min _{X_{N-1}}\left(d\left(f\left(S_{N-1}, X_{N-1}\right), S_{G}\right)\right) \\
= & \text { minimum error at } t=N \\
& \text { achievable from } S_{N-1} \\
e_{N-2}\left(S_{N-2}\right)= & \min _{X_{N-2}}\left(e_{N-1}\left(f\left(S_{N-2}, X_{N-2}\right)\right)\right) \\
= & \text { minimum error at } t=N \\
& \text { achievable from } S_{N-2} \\
\ldots & \\
e_{0}\left(S_{0}\right)= & \min X_{0}\left(e_{1}\left(f\left(S_{0}, X_{0}\right)\right)\right) .
\end{aligned}
$$

Then, proceeding in the forward direction we can determine the policy function by successively minimizing the error at times $t=0, \ldots, N$.

At this juncture, fuzzy-logic-based control is used extensively in a wide variety of consumer products and industrial systems ranging from cameras and washing machines to cement kilns and subways. In such applications, a fuzzy model of the system is not known. Instead, the point of departure is the designer's or operator's perception of the fuzzy rules which should be used to achieve a desired objective. Viewed in this perspective, in most of its current applications fuzzy control involves a representation of what is basically a human solution in the form of fuzzy if-then rules expressed in FDCL.

It should be realized, however, that a human solution is implicitly rooted in a fuzzy model of the system under control. Thus, the main difference between the prescriptive fuzzy-model-based approach described earlier and the descriptive 'model-free' approach which is employed in current practice reduces to the question of whether the fuzzy model is explicit or implicit. At this juncture, our understanding of how fuzzy models can be constructed and dealt with is still rather rudimentary. In coming years, as our understanding of fuzzy models improves, prescriptive approaches to fuzzy control are likely to gain in importance and visibility.

\section{Fuzzy system identification and induction of rules from observations}

In dealing with fuzzy rules, it is important to differentiate between the surface structure and the deep structure of a fuzzy rule.

The surface structure of a rule is the rule expressed in a symbolic form, e.g.,

$$
\text { if } \mathrm{X} \text { is } \mathrm{A} \text { then } \mathrm{Y} \text { is } \mathrm{B} \text {. }
$$


The deep structure of a rule is its surface structure together with the definitions of linguistic values which appear in its antecedent and consequent. In the case of the rule represented by (13), the deep structure is the rule itself together with a specification of the membership functions of $\mathrm{A}$ and $\mathrm{B}$.

The concepts of surface and deep structure provide a convenient point of departure for the formulation of the identification problem in the context of fuzzy rule-based systems.

The basic identification problem has two variants. In the first variant, the starting point is the knowledge of the surface structure of a system, $S$, which is the object of identification. The problem is to approximate to the deep structure of $\mathrm{S}$ based on given input-output data, that is, a collection of input-output pairs.

The second variant is considerably more difficult than the first. In this variant, the problem is the same but the surface structure is not known initially. A seminal contribution to the solution of this problem was made by Takagi and Sugeno (1985). Today, there is a fairly extensive literature on the problem in question but a definitive solution is not yet in hand. In what follows, we shall discuss very briefly the simpler (first variant) of the identification problem.

As is typical of standard approaches to the identification problem, the point of departure is the postulated structure of a fuzzy system modulo the parameters of linguistic variables in the fuzzy if-then-rules.

Many of the approaches described in the literature are based on variants of the Takagi-Sugeno-Kang (TSK) architecture, which is illustrated in Fig. 9. In this architecture, the input $X$ is assumed to have $k$ components $X_{1}, \ldots, X_{k}$ and the consequent of rule $R^{i}$ is assumed to be a linear combination of the constituents of $X$. The output, $Y$, is a convex combination of the consequents, with the weight, $w_{i}$, being the conjunction of the grades of membership of $X_{1}, \ldots, X_{k}$ in the antecedents $A_{1}^{i}, \ldots, A_{k}^{i}$, respectively. In a frequently employed variant of this architecture, $\wedge$ is replaced with * (product) and the membership functions are assumed to be triangular or trapezoidal. In

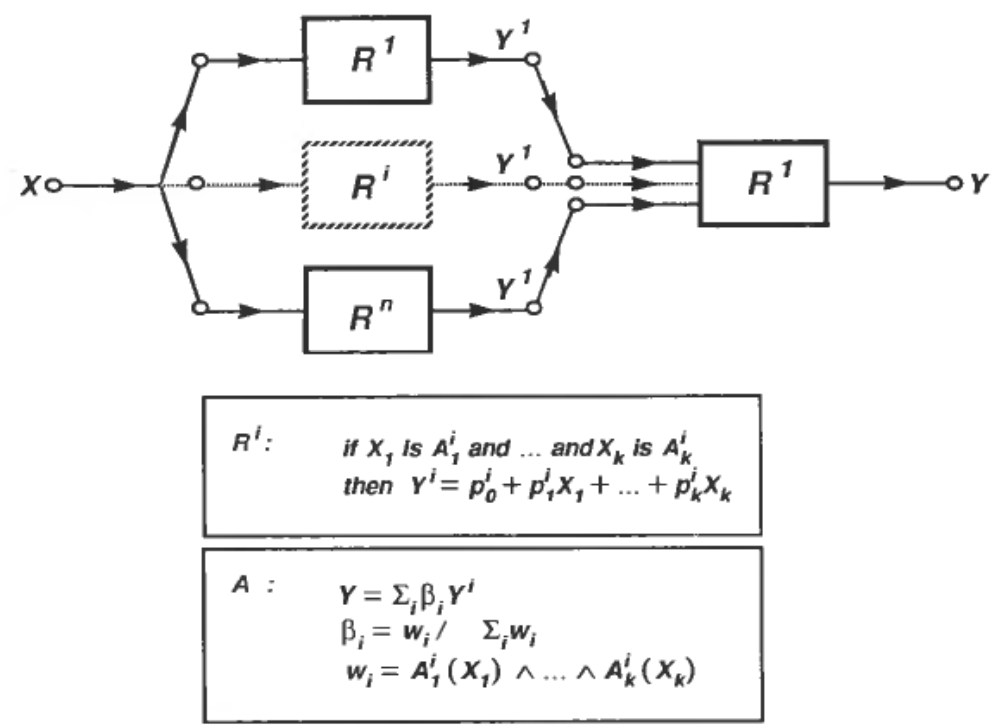

Figure 9. Takagi-Sugeno-Kang architecture. 


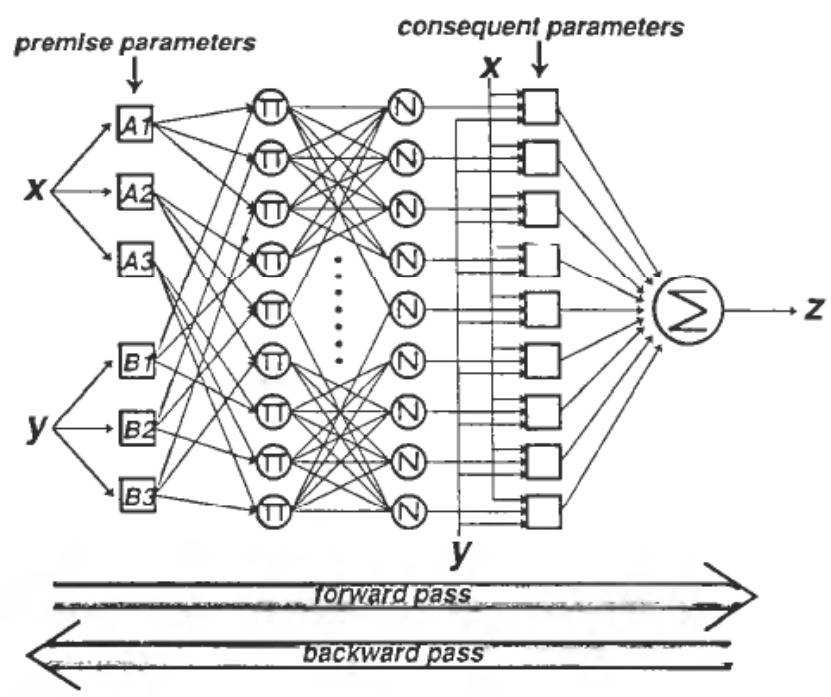

Figure 10. The Jang architecture. In the illustrated system, there are two inputs, $x$ and $y$, and three rules.

other variants, the membership functions are assumed to be gaussian. This assumption leads to methods which are similar to the radial basis function approaches used in neurocomputing (Warwick et al. 1992).

A basic idea underlying the approaches described in the literature is that a fuzzy system based on the TSK architecture can be represented as a multilayer feedforward network. In the Jang approach (Jang 1992), for example, the system is represented as shown in Fig. 10. The circles with $\Pi$ and $N$ represent multipliers and normalizers, respectively.

Representing a fuzzy system as a mulitayer structure lays the groundwork for the application of neurocomputing techniques and, in particular, the backpropagation algorithm (Hertz et al. 1991) to the determination of parameters of membership functions. In a more basic way, the techniques in question are rooted in dynamic programming (Werbos 1974) and, more particularly, in gradient programming, which through chain differentiation leads to the backpropagation algorithm (Zadeh 1994). More detailed expositions of these methods may be found in (Jang 1992, Lin and Lee 1991, Wang 1993 and Takagi 1991).

In addition to the use of techniques borrowed from neurocomputing, there are many promising approaches based on reinforcement learning (Berenji 1993) and genetic algorithms (Lee and Takagi 1994). Important progress had been made in our understanding of the identification problem in the context of fuzzy systems, but many difficult problems remain to be addressed.

\section{Concluding remarks}

In coming years, a significant paradigm shift in systems analysis and control is likely to take place. The shift in question involves a move toward the replacement of crisp foundations of modern control theory and systems analysis by a foundation based on fuzzy logic and soft computing - a collection of computing methodologies which are tolerant of imprecision, uncertainty and partial truth. 
In my perception, the shift in question will have an invigorating effect on control theory and systems analysis. It will open the door to the development of systems which will have a much higher MIQ (Machine Intelligence Quotient) than those which could be conceived and designed through the use of traditional methods.

\section{ReFERENCES AND Related PAPERS}

Bellman, R. E., and ZADEH, L. A. (1970). Decision-making in a fuzzy environment, Management Science, 17, B141-B164.

BERENII, H. R. (1991). Fuzzy logic controllers, In An Introduction to Fuzzy Logic Applications in Intelligent Systems, edited by R. R. Yager and L. A. Zadeh (Kluwer Academic Publishers), pp. 69-96.

Chang, S. S. L., and Zadeh, L. A. (1972). On fuzzy mapping and control, IEEE Trans. Systems, Man, and Cybernetics, 2, 30-34.

Driankov, D., Hellendoorn, H., and Reinfrank, M. (1993). An Introduction to Fuzzy Control (Springer-Verlag, Berlin).

Dubois, D., Prade, H., and YAGer, R. (Eds.)(1993). Readings in Fuzzy Sets for Intelligent Systems (Morgan Kaufmann, San Mateo).

Hertz, J., KroGH, A., and PAlmer, R. (1991). Introduction to the theory of neural computation (Addison Wesley).

JAmshidi, M., VAdieE, N. and Ross, T. (Eds.) (1993). Fuzzy Logic and Control (Prentice Hall, Englewood Cliffs, NJ).

JANG, J.-S. R. (1992). Self-learning fuzzy controller based on temporal back-propagation. IEEE Trans. on Neural Networks, 3, 714-723.

Kandel, A., and Langholz, G. (Eds.) (1994). Fuzzy Control Systems (CRC Press, Boca Raton).

KARR, C. (1991). Genetic algorithms for fuzzy controllers. AI Expert, 6, 26-33.

Kosko, B. (1991). Neural Networks and Fuzzy Systems: A Dynamical Systems Approach to Machine Intelligence (Englewood Cliffs, Prentice-Hall).

LEE, C. C. (1990). Fuzzy logic in control systems: Fuzzy logic controller, Part I and Part II, IEEE Trans. Systems, Man and Cybernetics, 20, 404-418.

LEE, M. A. and TAKAGI, H. (1993). Integrating design stages of fuzzy systems using genetic algorithms. 2nd International Conference on Fuzzy Systems (FUZZ-IEEE '93), pp. 612617, March 28-April 1.

LIN, C.-T. and GeORGE LEE, C. S. (1991). Neural-network-based fuzzy logic control and decision system. IEEE Transactions on Computers, 40, 1320-1336.

Mamdani, E. H. and Assilian, S. (1975). An experiment in linguistic synthesis with a fuzzy logic controller. Int. J. Man-Machine Studies, 7, 1-13.

Pedrycz, W. (1989). Fuzzy Control and Fuzzy Systems (John Wiley, New York).

Sugeno, M. and Kang, G. T. (1988). Structure identification of fuzzy model. Fuzzy Sets and Systems, 28, 15-33.

Terano, T., Asal, K. and Sugeno, M. (1992). Fuzzy Systems Theory and its Applications (Academic Press).

TAKAGI, T. and SugEno, M. (1985). Fuzzy identification of systems and its applications to modeling and control, IEEE Trans. Systems, Man and Cybernetics, 15, 116-132.

Wang, L.-X. (1994). Adaptive Fuzzy Systems and Control: Design and Stability Analysis (PTR Prentice Hall, Englewood Clifts, New Jersey).

WARWICK, K., IRWIN, G. W. and HUNT, K. J. (1992). Neural networks for control and systems (London, Peter Peregrinus Ltd.).

Werbos, P. J. (1974). Beyond Regression: New Tools for Prediction and Analysis in the Behaviour Sciences, Ph.D. Thesis, Harvard University, Committee on Applied Mathematics.

ZADEH, L. A. (1971). Toward a theory of fuzzy systems, In Aspects of Network and System Theory, R. E. Kalman and N. DeClaris (eds.) (New York: Rinehart \& Winston), 469-490.

ZADEH, L. A. (1972). A rationale for fuzzy control, J. Dynamic Systems, Measurement and Control 94, Series G, 3-4.

ZADEH, L. A. (1973). Outline of a new approach to the analysis of complex systems and decision processes, IEEE Trans. Systems, Man and Cybernetics, 3, 2844.

ZADEH, L. A. (1974a). On the analysis of large scale systems, In Systems Approaches and Environment Problems, H. Gottinger(ed.), 23-27 (Gottingen, Vandenhoeck and Ruprecht). 
ZADEH, L. A. (1974b). A fuzzy-algorithmic approach to the definition of complex or imprecise concepts, Electronics Research Laboratory Report ERL-M474, University of California, Berkeley, (also published in Int. Man-Machine Studies, 8, 249-291, 1976).

ZADEH, L. A. (1975). The concept of a linguistic variable and its applications to approximate reasoning, I, Information Sciences, 8, 199-249.

ZaDEH, L. A. (1994). Fuzzy Logic, Neural Networks and Soft Computing, Comm. ACM, 37, 7784.

ZIMMERMAN, H. J. (1990). Fuzzy Set Theory and Its Applications, second edition(Kluwer-Nijhoff). 